\title{
On the cessation of magnetic reconnection
}

\author{
M. Hesse ${ }^{1}$ and J. Birn ${ }^{2}$ \\ ${ }^{1}$ Code 696, NASA Goddard Space Flight Center, Greenbelt, MD, 20771, USA \\ ${ }^{2}$ NIS-1, Los Alamos National Laboratory, Los Alamos, NM, 87545, USA
}

Received: 11 March 2003 - Revised: 17 June 2003 - Accepted: 23 June 2003 - Published: 1 January 2004

\begin{abstract}
Kinetic simulations of collisionless magnetic reconnection are used to study the effect on the reconnection rate of ion density enhancements in the inflow region. The goal of the investigation is to study a candidate mechanism for the slow-down of magnetic reconnection. The calculations involve either proton or oxygen additions in the inflow region, initially located at two distances from the current sheet. Protons are found to be much more tightly coupled into the evolution of the reconnecting system and, therefore, they effect an immediate slowdown of the reconnection process, as soon as the flux tubes they reside on become involved. Oxygen, on the other hand, has, within the limits of the calculations, a much less pronounced effect on the reconnection electric field. The difference is attributed to the lack of tight coupling to the magnetic field of the oxygen populations. Last, a study of proton and oxygen acceleration finds that protons respond primarily to the reconnection electric field, whereas the main oxygen electric field is achieved by Hall-type electric fields at the plasma sheet boundary.
\end{abstract}

Key words. Space plasma physics (magnetic reconnection; numerical simulation studies; numerical simulation studies)

\section{Introduction}

Magnetic reconnection, or merging, is the dominant mechanism that facilitates plasma transport across boundaries by means of a localized violation of the frozen magnetic flux condition (Axford, 1984). Thus, magnetic reconnection has been recognized as a prime transport process in as diverse environments as the solar atmosphere (e.g. Priest, 1981), the magnetopause (e.g. Russell and Elphic, 1979; Sonnerup et al., 1981) and nightside current sheet of the Earth's magnetosphere (e.g. Hones, 1977; Baker et al., 1996), astrophysical plasmas (e.g. Schindler et al., 1991), and laboratory experiments (e.g. Yamada et al., 1997).

Correspondence to: M. Hesse (michael.hesse@nasa.gov)
Since the first description of magnetic reconnection by Giovanelli (1946), much has been learned about the role reconnection plays in plasma dynamics. For example, reconnection processes are essential in the energy entry into the magnetosphere (Perrault and Akasofu, 1978; Mozer et al., 2002), and then they control the distribution and conversion of energy within the magnetosphere (Hesse, 1995). Specifically, modern research based on Geotail spacecraft observations leaves little doubt that magnetic reconnection is the main engine of substorm evolution in the magnetosphere (Machida et al., 2000; Nagai et al., 1998).

Recent analyses, based on both observations and modeling, have also begun to focus on the inner dissipation region, where particles become unmagnetized and change magnetic flux tubes (Vasyliunas, 1975). For example, a number of simulations have shown that quadrupolar magnetic field signatures form if nearly antiparallel magnetic fields reconnect (e.g. Drake and Mand, 1994; Hesse and Winske, 1994), a feature suggested earlier based on theoretical arguments (Sonnerup et al., 1981), and found recently in spacecraftbased observations (Nagai et al., 2001; Øieroset et al; 2001). Recent theoretical and modeling activities proceeded even further to look at electron dynamics in the inner diffusion region (Horiuchi and Sato, 1994; Hesse et al., 1999; Pritchett, 2001). Here, pressure anisotropies generated by electron bounce motions appear to be a critical mechanism in nearantiparallel reconnection. More complex processes appear to be involved in component merging, where the magnetic field rotates through less than 180 degrees (Hesse et al., 2002; Drake et al., 2003).

Thus, through a combination of spacecraft observations, theory, and modeling, we are beginning to understand how magnetic reconnection operates. It is well known, however, that reconnection often appears to operate in a bursty fashion, or ceases activity after some time. Examples are to be found in the solar atmosphere, where eruptive processes are time-limited (e.g. Priest and Forbes, 2002), and at the magnetopause of the Earth, where flux transfer events (FTEs) are signatures of a temporally and spatially limited reconnection 
process (Russell and Elphic, 1979). Furthermore, it is a wellknown observational fact that the magnetic flux in the magnetospheric lobes remains finite at the end of substorm activity (e.g. Pulkkinen et al., 1991). This indicates that magnetic reconnection ceases before the magnetic flux reservoir in the inflow region is exhausted. In addition, observations of bursty bulk flows (Angelopoulos et al., 1992), potentially the nightside analogue of FTEs, strongly support the notion of bursty reconnection.

Because the operation and energy conversion time of magnetic reconnection determines the total amount of mass, momentum, magnetic flux, and energy transport, an understanding of why magnetic reconnection ceases is of basic importance to the description of collisionless plasma systems. The present paper attempts to shed light on a possible mechanism for the cessation of magnetic reconnection, a drastic increase in the plasma density in the inflow region. The study is motivated by the fact that magnetic reconnection is strongly influenced by the properties of the plasma upstream of the reconnection site, which affect the properties at the reconnection site itself. For instance, changes in the plasma source region from a dense plasma sheet to tenuous lobe plasma have been found to accelerate the reconnection rate, at least within MHD simulations of magnetotail reconnection (Hesse et al., 1996). Hence, changes in the source populations from tenuous lobe plasma to denser plasma might be expected to reduce the reconnection rate drastically, or even suppress reconnection. This change in the source population could be related to the presence of oxygen inside the magnetotail lobes (e.g. Baker et al., 1982), as well as the presence of the plasma mantle at farther distances down the magnetotail (e.g. Gosling et al., 1984).

\section{The model}

For the purpose of the present study, we employ particle-incell simulations, which treat both ions and electrons kinetically. The simulations utilize our self-consistent particlein-cell simulation code (Hesse et al., 1999), which has been applied to a variety of reconnection problems (e.g. Hesse et al., 2001), as well as to studies of anisotropy-driven instabilities (Gary et al., 2000). We focus on anti-parallel merging, as reconnection on the nightside of the magnetosphere predominantly involves nearly anti-parallel fields, and even recent magnetopause studies indicate that antiparallel reconnection may dominate over component merging (Le et al., 2001).

Recent results indicate that three-dimensional effects remain very limited in anti-parallel reconnection (Hesse et al., 2001; Zeiler et al., 2002), such that the system behaves mostly like a translationally invariant sheet. This allows us to restrict, for simplicity, the calculation to 2.5-dimensional calculations. We normalize the calculation in the following way. Lengths are normalized to the ion inertial length, $c / \omega_{i}$, and time is normalized to the inverse of the ion cyclotron period, $\Omega_{i}$. Number densities are normalized to the maximum density of current carrying particles, $n_{0}$. Finally, velocities are normalized to the (proton) Alfvén speed, $v_{A}$, mass is normalized to the proton mass $m_{p}$, and the magnetic field is normalized to the asymptotic value in the inflow region, $B_{0}$.

In addition, we further simplify the analysis by investigating the same configuration as studied during the GEM reconnection challenge (Birn et al., 2001), with the addition of a background population. The GEM problem was defined as follows: a Harris-sheet solution (Harris, 1962), with a sheet thickness of $L=0.5$ was integrated in a box of $0<x<25.6 ;|z|<6.4$. We note that a thickness of $L=0.5$ is smaller than quiet time current sheet widths, but quite compatible with observed thin current sheet scales of a few hundred km (e.g. Sanny et al., 1994; Hoshino et al., 1996). A constant density of $n_{b}=0.2$ (same temperature as the plasma sheet population) is added to the Harris-sheet density distribution. We will, in the following, refer to this combined proton density as "foreground density." This density prevents reconnection from proceeding into the vacuum limit and leads, in the absence of any further populations, to an asymptotic reconnection electric field of $E \approx 0.24$ (Birn et al., 2001). A finite-amplitude magnetic field perturbation to the magnetic field generates a normal magnetic field of some 3\% amplitude in the current sheet.

In the present investigation, we add to these parameters additional cold background populations, consisting of either protons or oxygen, in the inflow region. The goal is to study the impact these populations may have on the reconnection rate, which was previously determined by the combination of lobe magnetic field strength and foreground density. The number densities for these additional populations are chosen to lead to equal mass densities (of 1.6), and thus, to equal Alfvén velocities. For protons and oxygen, this implies number densities of 1.6 and 0.1 , respectively. The background populations are added at two distances from the current sheet center. Figure 1 displays the initial distribution of background particles, as a function of $z$, for all runs. The black graph shows the foreground proton distribution, whereas the red and blue graphs indicate the distribution of background mass densities, which are initially restricted to $|z|>1.5$ or $|z|>2.5$, respectively.

All calculations use a proton/electron mass ratio of $m_{p} / m_{e}=100$. The foreground proton/electron temperature ratio is $T_{i} / T_{e}=5$, and $\omega_{e} / \Omega_{e}=2$. Approximately $1 \times 10^{8}$ macroparticles are employed, and a grid of $n_{x} \times n_{z}=800 \times 400$ is used for the integration with a time step of $\omega_{e} \delta t=1$. A total of five runs is performed: a run without background population, and one run each for proton and oxygen backgrounds, at $|z|>1.5$ or $|z|>2.5$.

\section{Results: effects on the evolution}

The reconnected magnetic flux, defined by

$$
F=\int_{z=0}\left|B_{z}\right| d x,
$$




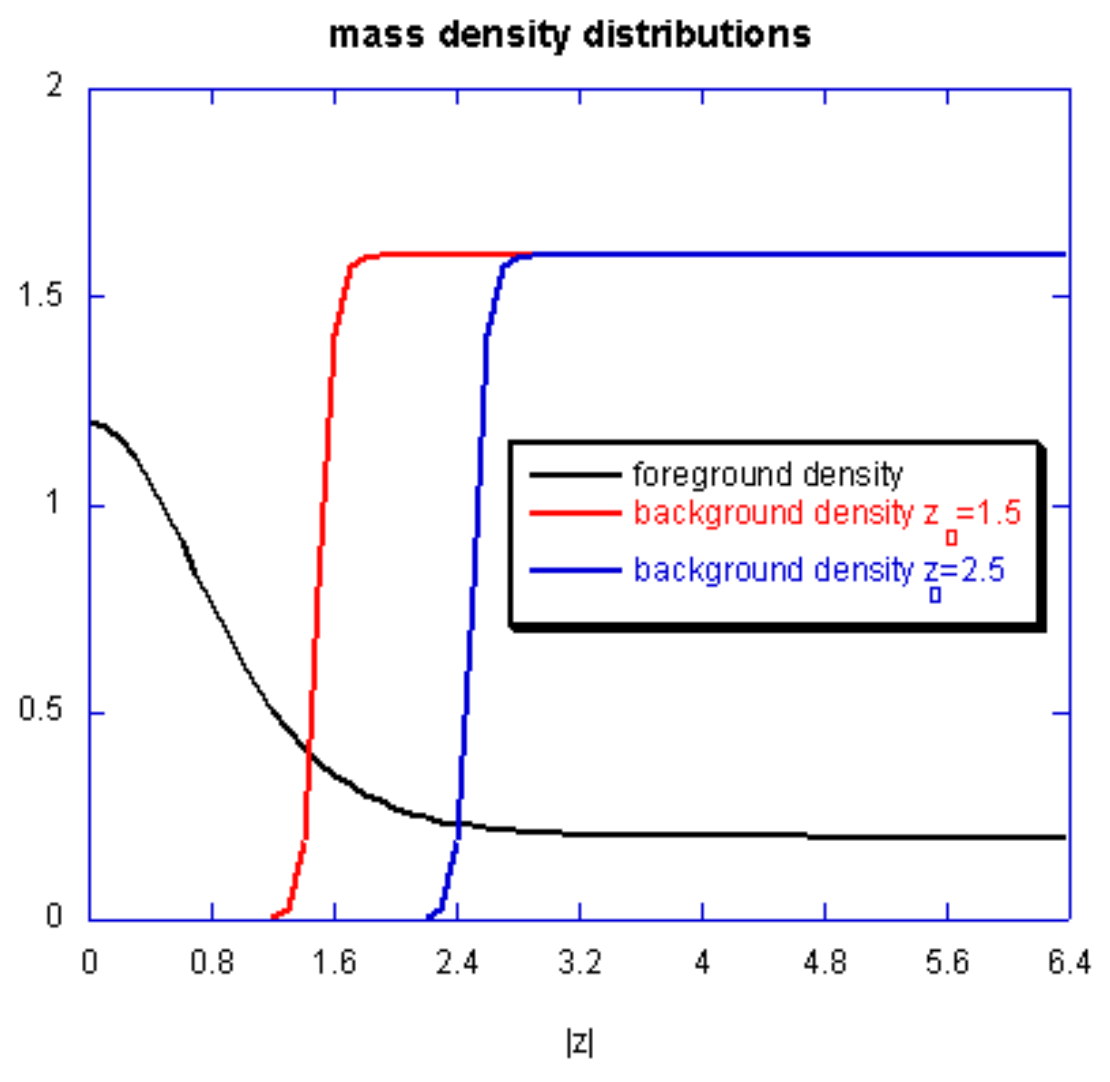

Fig. 1. Plot of the initial mass density distribution. The foreground hot plasma density is denoted by the black curve. Background mass densities of either oxygen or protons are added at the locations indicated by the blue and red graphs. where the integral is taken between $X$ and $O$ points, provides a measure of the overall progress of the evolution. It is shown in Fig. 2, for all five runs discussed here. Figure 2 also displays the time evolution of the reconnection electric field. The figure shows that all simulations start with very similar growth rates, identical to the rates found in earlier calculations (Birn et al., 2001). This indicates that the reconnection process initially only involves the immediate neighborhood of the diffusion region. For times $t>22$, the runs without background show a reduction of reconnection rate, due to the exhaustion of magnetic energy in the inflow region and the backpressure generated by the magnetic island. At times $t>10$, the evolution for the run with the proton background at $|z|>1.5$ begins to deviate from the others, featuring a strongly reduced reconnection rate. A similar reduction begins for $t>14$ for the run with proton background at $|z|>2.5$. Thus, proton backgrounds substantially influence the reconnection rate.

Surprisingly, both runs with oxygen background do not show any significant reduction of the reconnection rate, despite initial mass density distributions identical to those of the protons. This result is confirmed by Fig. 3, which displays, for $t=20$, magnetic fields and current densities (top panels) and background number densities (bottom panels) for both oxygen and proton backgrounds, initially located at $|z|>2.5$. A comparison of top and bottom panels leads to very different results. While the magnetic field and current density evolution is clearly further advanced for back- ground oxygen, the proton background has changed considerably further from its initial distribution. Thus, we conclude that background oxygen does not strongly restrict the reconnection rate, and does not become strongly involved in the evolution. A proton background, on the other hand, strongly participates in the evolution, which slows down substantially as a result.

As a step toward explaining this phenomenon, Fig. 4 displays the foreground and background charge densities for both calculations, which feature backgrounds initially at $|z|>2.5$. The left column displays the effects of the oxygen background, and the right column displays the ones of the proton background. A comparison between the panels shows that the charge density of the proton background far exceeds that of the oxygen background in the lower left panel. Ion charge densities tie electron charge densities, which, are closely tied to the magnetic field in the inflow region. Thus, in the case of the oxygen background, a relatively small charge density perturbation can be compensated for by foreground electron redistribution along magnetic field lines. This, however, is not possible in case of the proton background. Here, too many electrons are bound to the background population, such that the latter needs to move in order for the magnetic field to change topology. Because of the background mass, this process cannot proceed as fast as in the oxygen calculations. 

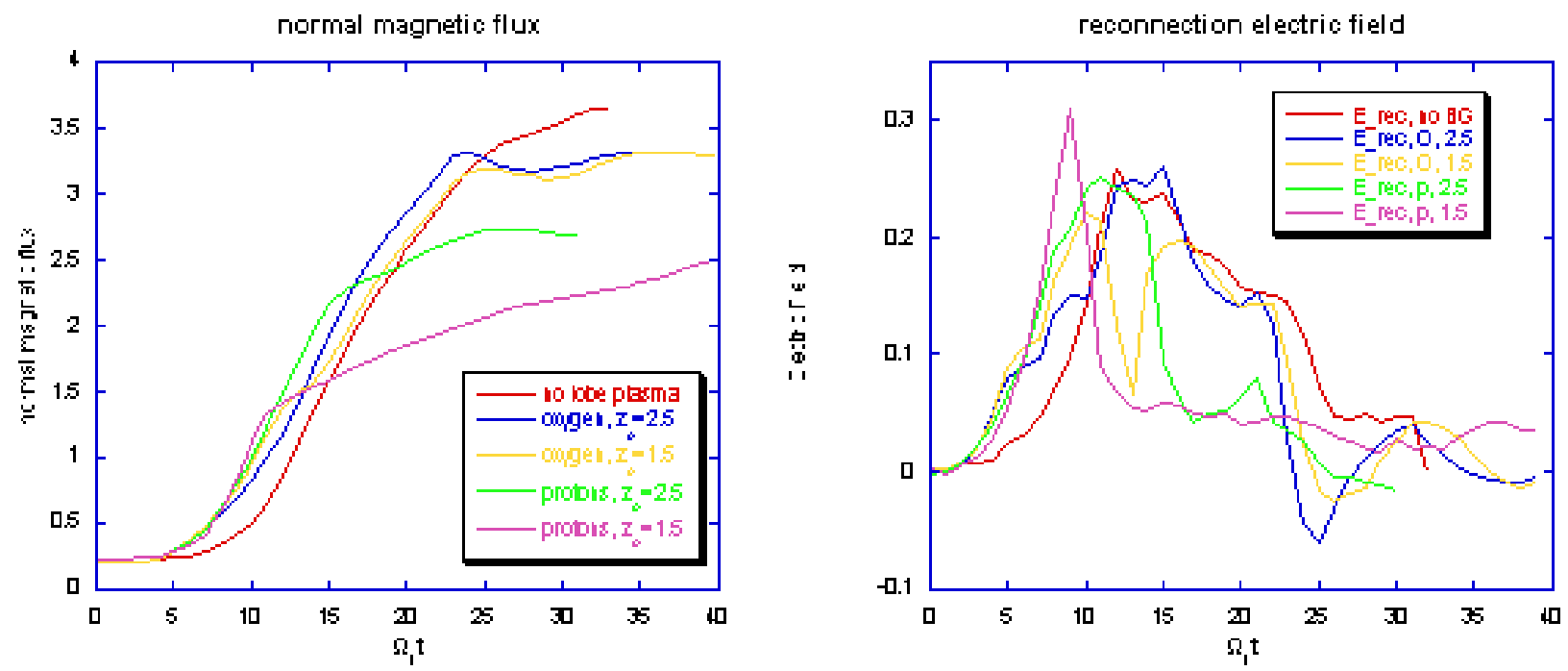

Fig. 2. Time evolution of the magnetic flux threading the current sheet (left panel), and reconnection electric field (right panel). Different graphs are for different runs. The evolution of magnetic reconnection is nearly identical for the runs involving background oxygen and the run without any background populations. Depending on the distribution of background protons, those runs feature strong reductions in the reconnection rate.

\section{Oxygen, $\mathrm{z}_{0}=2.5 \quad \Omega_{\mathrm{i}} \mathrm{t}=\mathbf{2 0}$ Protons, $\mathrm{z}_{0}=2.5$}
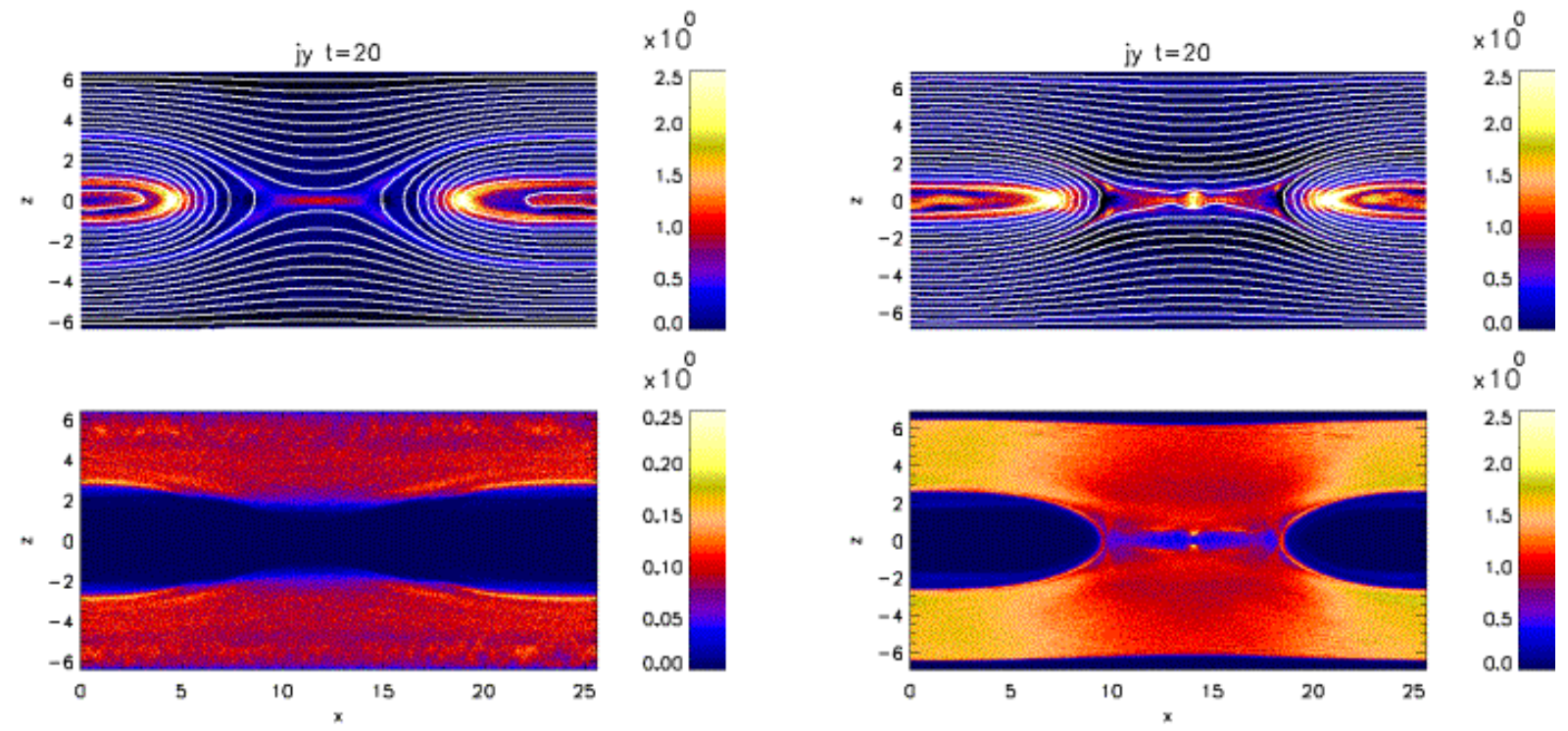

$\times 10^{\circ}$

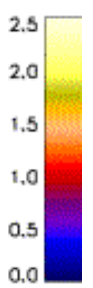

Fig. 3. Comparison of magnetic fields and currents (top panels) and background populations densities for two runs. The runs involve either oxygen (left) or proton (right) background populations, added at $|z|>2.5$.

\section{Results: background acceleration}

The second problem in need of answering concerns the mechanism, which accelerates background particles, such that they may (or may not) follow the overall evolution of the magnetic field lines. As the dominant mechanism differs for oxygen and proton backgrounds, we will discuss each mechanism in turn.
The main contributions to the acceleration of background oxygen are shown in Fig. 5. The left panels describe the effects of the acceleration, which took place prior to the $t=22$, the time shown. The top left panel shows the oxygen bulk flow speed. The plot features a double structure of strongly enhanced flow toward the center of the current sheet. The lower panel displays the total particle kinetic energy of the oxygen population. The pattern is similar to the one of the velocity, with larger energy density further away from the 


\section{Ion charge densities $\Omega_{\mathrm{i}} \mathbf{t}=\mathbf{2 0}$}
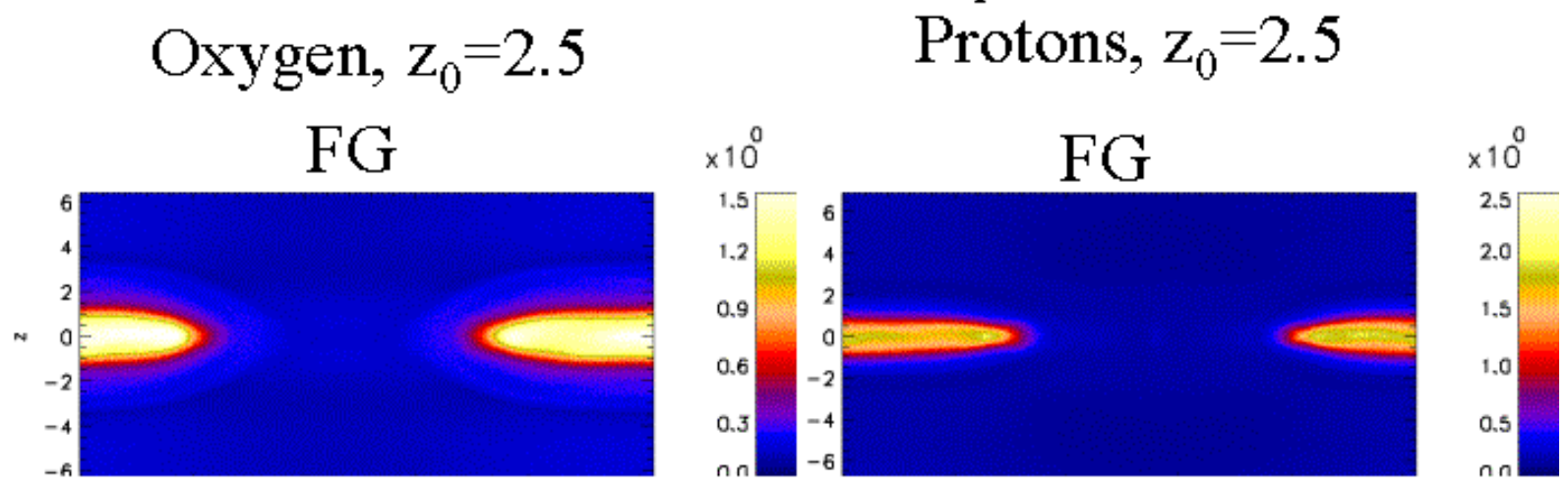

\section{BG/oxygen}
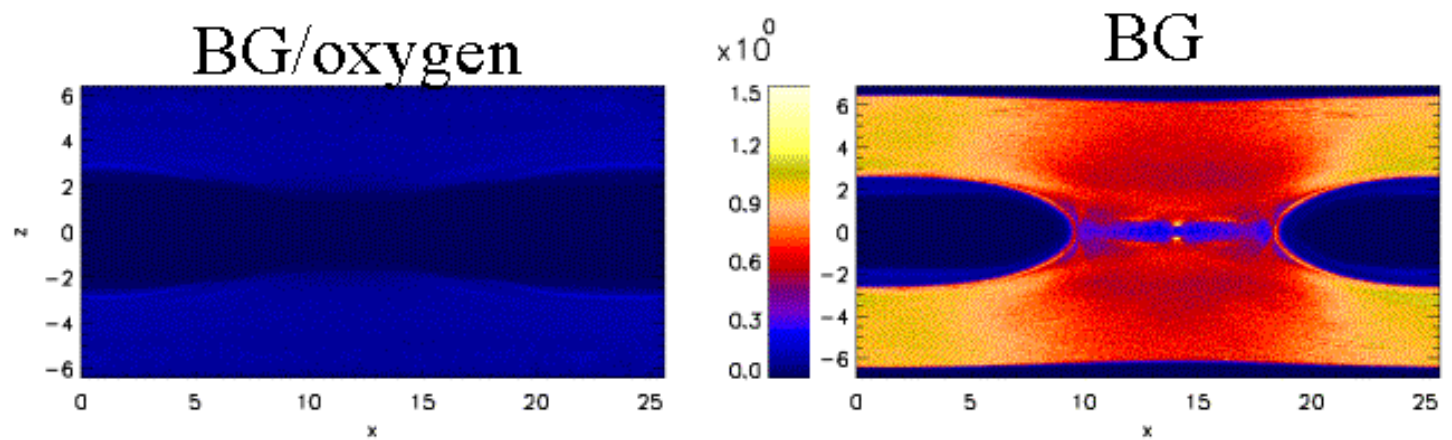

\section{$\times 10^{0}$}

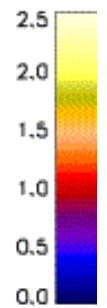

Fig. 4. Foreground (top) and background (bottom) charge densities for the same runs of Fig. 3.

\section{Oxygen Acceleration}

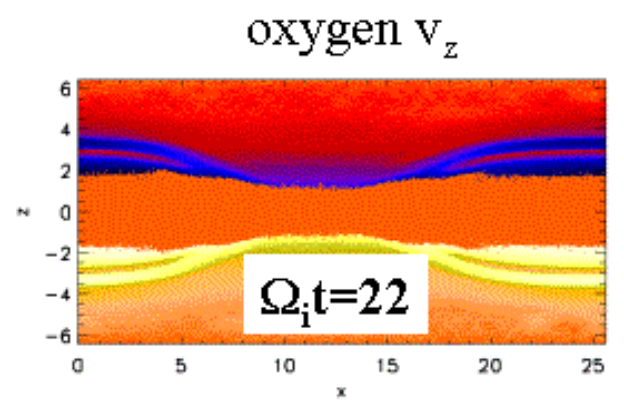

oxygen kinetic energy density

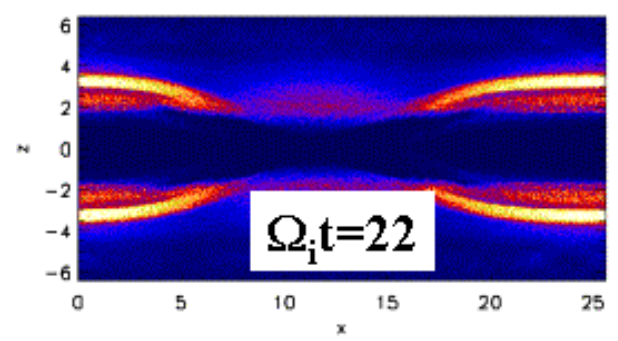

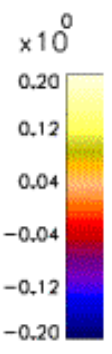
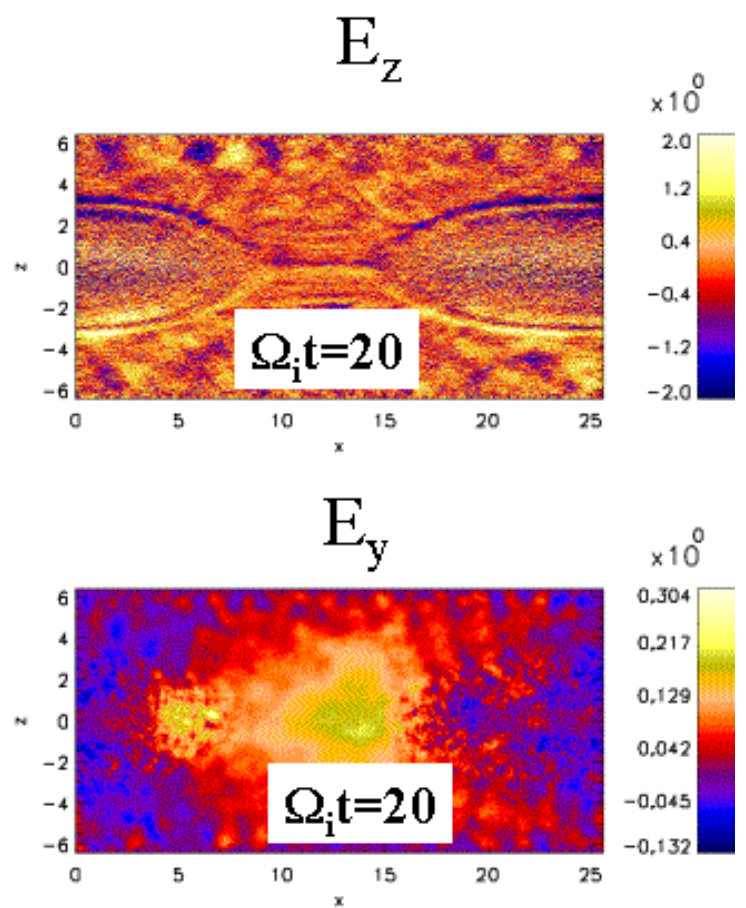

Fig. 5. Oxygen acceleration: $z$ component of the oxygen background velocity (top left), $z$ component of the electric field (top right), total oxygen kinetic energy density (bottom left), and $y$ component of the electric field (bottom right). 
y component oxygen velocity, $t=22$

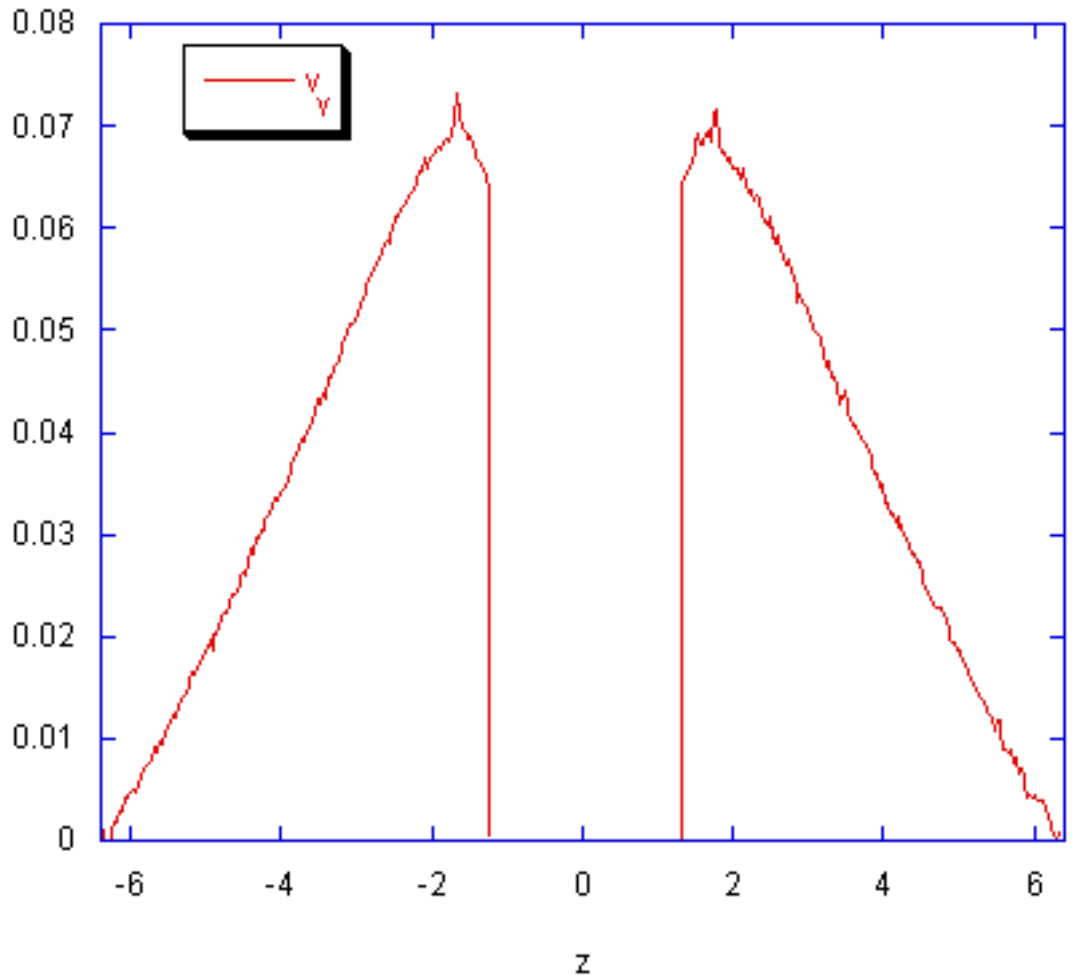

Fig. 6. The $y$ component of the oxygen velocity, taken at $x=12.8$ and $t=22$. current layer, i.e. in regions of higher number density. This energy distribution demonstrates that energy deposition is exclusively in the form of bulk flow, i.e. the background population is heated negligibly. The panels on the right display the two electric field components primarily involved in the oxygen acceleration process: $E_{z}$ and $E_{y}$. Both are taken at $t=20$, slightly earlier than the panels on the left. Comparing $E_{z}$ with both of the left panels shows an exact match of patterns of enhanced $E_{z}$ with patterns of enhanced $v_{z}$ of the oxygen, demonstrating an essential role of the $E_{z}$ component of the electric field. The acceleration by the $E_{z}$ component of the electric field is mainly ballistic: for each individual oxygen particle, acceleration in an electric field over a distance $\delta z$ yields a final velocity of

$v=\sqrt{\frac{2 E_{z} \Delta z}{m},}$

if the initial velocity is negligible. Using values of $E_{z} \approx 1$ and $\Delta z \approx 0.5$ leads to a final velocity of $v \approx 0.25$, very close to what is shown in the top left panel for Fig. 5. This analysis shows that oxygen acceleration is primarily accomplished by the interaction of Hall-type electric fields, generated by the primary reconnection dynamics (e.g. Mandt et al., 1994), with the cold oxygen particles. Owing to the comparatively low charge density represented by the oxygen in the present calculation, oxygen particles act mainly as test particles, which are effected by the electromagnetic fields associated with the reconnection process.
Acceleration by the $y$ component of the electric field is considerably less effective. Here, acceleration is primarily in the $y$ direction, by the reconnection electric field. Assuming that cold particles do not move much unless exposed to an electric field, the final velocity is determined by

$v_{y}=\frac{1}{m} \int E_{y} d t$.

Figure 2 provides an estimate for the time-integral of $E_{y}$. The reconnected flux is, in our normalization, identical to the value of the time integral of $E_{y}$ at the center of the reconnection region. At $t=22$, the present run leads to an estimated velocity of $v_{y} \approx 0.16$. This value is higher by a factor of two than the velocities shown in Fig. 6, which displays a plot of the $y$ component of the oxygen velocity along $x=12.8$. This difference is attributable to the action of the Lorentz-force, provided by the $B_{x}$ component of the magnetic field, on accelerated oxygen ions. It should be noted that $t=22$ is just in excess of an oxygen Larmor period. Due to the time variation of $E_{y}$, however, oxygen ions typically complete only a fraction of a full Larmor orbit during the simulated interval. The enhanced $v_{z}$ velocity, seen in the top left panel of Fig. 5, near the center (in $x$ ) of the simulation box, is, therefore, due to the action of $E_{y}$, followed by the action of the magnetic field in the inflow region.

The acceleration of protons is shown in Fig. 7, in the same format as Fig. 5. In the case of protons in the inflow region, the acceleration process of the background proceeds quite differently. During the progress of reconnection, pro- 


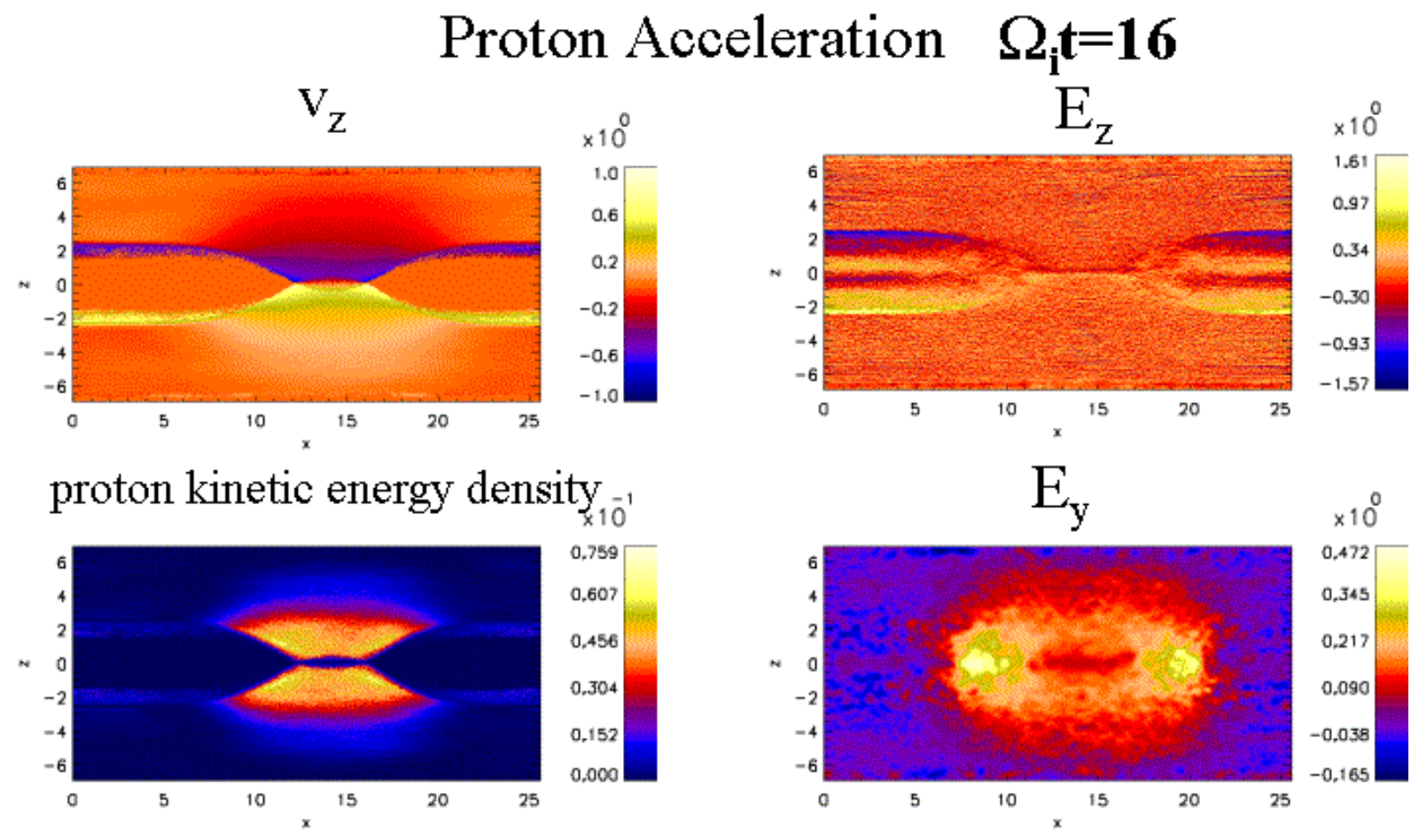

Fig. 7. Background proton acceleration, in the same format as Fig. 5.

tons execute many cyclotron orbits. Thus, the action of an electric field should lead primarily to a net $\boldsymbol{E} \times \boldsymbol{B}$ drift, unless spatial scales are much smaller than proton Larmor radii. Hence, the $v_{z}$ component of the proton velocity in the upper left panel is strongest in the center of the simulation region, where the $y$ component of the electric field is strongest and the magnetic field weakest. The proton kinetic energy enhancement shown in Fig. 7 demonstrates that the $E_{y}$-induced $\boldsymbol{E} \times \boldsymbol{B}$ drift also involves some proton heating. The resulting temperature is $T_{b g}=0.03$, which is small compared to the foreground temperature of $T_{f g}=0.5$.

In light of the comparatively rapid proton cyclotron motion, it might seem surprising that the enhancements of $v_{z}$, are roughly collocated with the increases of $E_{z}$. Figure 8 explains this effect. The Hall electric field is generated primarily by the evolution of the current-carrying, hot foreground plasmas. As reconnection expands to flux tubes originally located further away from the current sheet, the localized Hall electric fields propagate away from the current sheet, and eventually into the background population. The first reaction of the background population to the Hall electric field is by acceleration in the $z$ direction. Within a gyro-period, this acceleration is turned into an effective $\boldsymbol{E} \times \boldsymbol{B}$ motion by the proton gyro-orbit. Thus, the velocity pattern should feature enhanced $v_{z}$ close to the edge of the propagating electric field front, and enhanced $v_{y}$ further behind. This exact pattern is seen in Fig. 8. The front of the electric field is located at $z \approx 2.3$. The immediate reaction is an enhancement of $v_{z}$, followed by $v_{y}$ (at smaller values of $z$ ).
Clearly, the effectiveness of this process depends on the speed of propagation of the electric field front. In the present calculation, this propagation velocity is $v_{E} \approx 0.1-0.2$. This fairly rapid speed, combined with the narrow peak of the electric field distribution of $\Delta z \approx 0.3$, emphasizes transient behavior and the enhancement of $v_{z}$. For slower propagation and slower reconnection rates, less of $v_{z}$ should be seen with stronger $v_{y}$.

In summary, oxygen and proton background ions are accelerated by both $E_{y}$ and $E_{z}$. Owing to the substantial difference in mass, the primary acceleration process for oxygen is provided by the Hall electric fields associated with currents at the edge of the reconnecting plasma sheet. The reconnection electric field has a much lower impact. For protons, the relevance is reversed. The primary acceleration process is provided by the reconnection electric field $\left(E_{y}\right)$, which generates a rapid drift toward the center of the current sheet. Hall electric fields lead to a combination of transient enhancements of $v_{z}$, and drifts in the $y$ direction.

\section{Summary and discussion}

The major difference between the steady-state and bursty mode of magnetic reconnection is that merging ceases in one but not the other. Mechanisms to suppress magnetic reconnection fall into two categories: internal to the reconnection process, or external. For the former, it is conceivable that the electron physics in the inner dissipation region may be unable to support the reconnection rate required by the ex- 


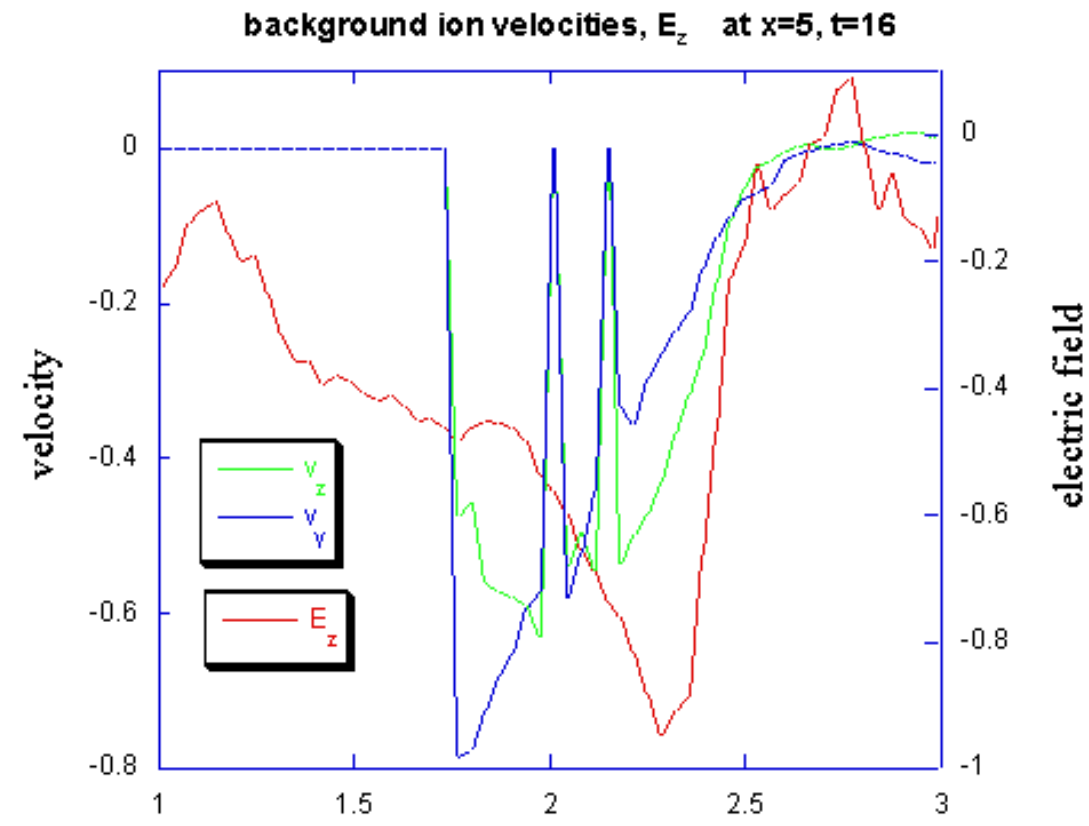

Z
Fig. 8. Background proton velocities at $x=5$ and $t=16$. Plotted are the $y$ and $z$-components of the proton velocity (blue and green, respectively), and the z-component of the electric field (red). ternal dynamics. In the absence of external driving, a more likely scenario has external conditions changing. A number of such candidate mechanisms may effect the cessation of magnetic reconnection. Among these are an exhaustion of energy supply, for example, if too much magnetic flux has been reconnected in a finite-size system, backpressure from reconnected flux tubes, such as the caused by magnetic field dipolarization in the magnetotail of the Earth (Baumjohann et al., 1999), or a change in inflow conditions. The present paper addresses the latter scenario.

More specifically, we performed a number of simulations with different plasmas in the inflow region of the magnetic reconnection process. In order to clearly identify the effects of those plasmas on the overall evolution, we deliberately chose in a well-understood, simplified geometry, provided by the GEM reconnection challenge (Birn et al., 2001). Simulating the same system, with the addition of plasmas in the inflow region, permits easy discrimination of any new effects introduced by the new populations. We performed a total of five simulations. The first baseline run was identical to the GEM problem (Hesse et al., 2001), while the others had additional cold plasma, either oxygen or protons, introduced at $|z| \geq 1.5$ or $|z| \geq 2.5$. Number densities of oxygen and proton background populations were chosen to lead to equal mass densities, and thus, equal Alfvén speeds. Thus, we expected a similar behavior, irrespective of particle species.

The simulations showed strong differences. First, the oxygen background appeared to have very little effect on the reconnection process, while a proton background led to a strong slowdown of the reconnection rate when the cold dense background population became involved in reconnec- tion. This occurred at $t=9$ for protons initially at $|z| \geq 1.5$ and at $t=13$ for protons initially at $|z| \geq 2.5$. Both cases showed a similar slowdown with a reconnection rate of less than a quarter of that found in the baseline run. This reduction is in excess of what would be expected based on the inflow Alfvén speed, which changes by a factor of only 2.5 during the evolution.

We found the cause of the difference between proton and oxygen backgrounds to be the different charge densities associated with either species. For equal mass densities, the proton charge density is sixteen times that of the (singly charged) oxygen. Accordingly, the proton background ties up a much larger number of highly magnetized electrons for overall charge neutrality. For proton backgrounds, these electrons constitute a major perturbation of the foreground charge density. Hence, the motion of magnetic flux tubes induced by the reconnection process requires that these electrons move as well. The electrostatic coupling, in turn, ties the electron motion to the motion of the background protons. The strong coupling into the overall dynamics of the background population mass generates a reduction in the reconnection rate.

Oxygen ions, on the other hand, constrained much fewer electrons, such that shifts in the overwhelming foreground electron density could compensate for much of the flux tube motion required for magnetic reconnection. At very late times, background oxygen ions became more affected. At those very late times, however, the reconnection rate suffered already from exhaustion of magnetic flux in the inflow region, and from the backpressure of the large magnetic island. 
We proceeded to a study of the acceleration mechanisms involved in generating the background particle motion during the simulations. The two electric field components immediately associated with the acceleration are the reconnection electric field $E_{y}$ (concentrated in the vicinity of the reconnection site), and the Hall electric field $E_{z}$, which forms at the edge of the plasma sheet and impacts foreground particle motion in the current direction between ions and electrons (Hesse et al., 1997). Here, again, we found a quite different response to this electric field for oxygen and protons. Oxygen was found to be immediately accelerated in a ballistic way when the Hall-electric field proceeded into the background population. The reconnection electric field, on the other hand, had a much smaller impact. Acceleration by $E_{y}$ primarily led to a motion in the current direction. An even lower velocity increase toward the current sheet resulted from the Lorentz force exerted by the magnetic field in the inflow region. Owing to its large mass, oxygen ions were unable to assume an $\boldsymbol{E} \times \boldsymbol{B}$ drift motion, driven by the reconnection electric field.

Background protons, on the other hand, featured the opposite behavior. Acceleration by the reconnection electric field immediately turned into an $\boldsymbol{E} \times \boldsymbol{B}$ drift motion by the rapid cyclotron motion associated with the low proton mass. Therefore, protons very quickly began to move toward the current sheet. In addition, Hall electric fields also affected background protons. Resulting enhancements of proton flow velocities toward the current sheet could be attributed to the transient effect of the moving Hall electric field enhancements. The major impact, however, was found to be an $\boldsymbol{E} \times \boldsymbol{B}$ drift in the $y$ direction.

Our simulations demonstrate that, on short time scales and kinetic spatial scales, the reconnection rate is not simply determined by the Alfvén speed in the inflow region. Instead, the rate reduction appears to be species-dependent. A strong proton density enhancement in the reconnection inflow region had the expected effect: a substantial reduction in the reconnection rate. Within the limitations of the present calculations, this effect was not found for oxygen. Due to the limited size and limited time interval of the simulated problem, we cannot conclude that oxygen will never have any effect on the reconnection rate. Also, the slower reconnection rates associated with thicker current sheets (Hesse et al., 2001a) may involve more pronounced oxygen effects. In these situations, it is likely that large enough displacements of magnetic flux tubes, such as are possible in larger systems, will require substantial oxygen motion and thus, ultimately a slow-down in the reconnection process. While our study is a first step only, the results do suggest, however, that very fast bursts of reconnection may terminate by density increases in tightly coupled ions in the inflow region.

Acknowledgements. Topical Editor T. Pulkkinen thanks E. Priest and another referee for their help in evaluating this paper.

\section{References}

Angelopoulos, V., Baumjohann, W., Kennel, C. F., Coronti, F. V., Kivelson, M. G., Pellat, R., Walker, R. J., Luehr, H., Paschmann, G.: Bursty bulk flows in the inner central plasma sheet, J. Geophys. Res., 97, 4027, 1992.

Axford, W. I.: Magnetic Reconnection, in: Magnetic Reconnection in Space and Astrophysical Plasmas, Geophys. Monogr. Ser., vol. 30, edited by Hones, Jr., E. W., p. 1, AGU, Washington D.C., 1984.

Baker, D. N., Hones, Jr., E. W., Young, D. T., and Birn, J.: The possible role of ionospheric oxygen in the initiation and development of plasma sheet instabilities, Geophys. Res. Lett., 9, 1337, 1982.

Baker, D. N., Pulkkinen, T. I., Angelopoulos, V., Baumjohann, W., and McPherron, R. L.: Neutral line model of substorms: Past results and present view, J. Geopys. Res., 101, 12975, 1996.

Baumjohann, W., Hesse, M., Kokubun, S., Mukai, T., and Petrukovich, A.: Substorm dipolarization and recovery, J. Geophys. Res., 104, 24 995, 1999.

Birn, J., Drake, J. F., M. A. Shay, M. A., Rogers, B. N., Denton, R. E., Hesse, M., Kuznetsova, M., Ma, Z. W., Bhattacharjee, A., Otto, A., and Pritchett, P. L.: GEM magnetic reconnection challenge, J. Geophys. Res., 106, 3715, 2001.

Drake, J. F. and Mandt, M. E.: Structure of thin current layers: implications for magnetic reconnection, Geophys. Res. Lett., 73, $1251,1994$.

Drake, J. F., Swisdak, M., Cattell, C., Shay, M. A., Rogers, B. N., and Zeiler, A.: Formation of electron holes and particle energization during magnetic reconnection, Science, 299, 873, 2003.

Gary, S. P., Winske, D., and Hesse, M.: Electron temperature anisotropy instabilities: Computer simulations, J. Geophys. Res., 105, $10751,2000$.

Giovanneli, R. G.: A theory of chromospheric flares, Nature, 158, 81,1946

Gosling, J. T., Baker, D. N., Bame, S. J., Hones, E. W., McComas, D. J., Zwickl, R. D., Slavin, J. A., Smith, E. J., and Tsurutani, B. T.: Plasma Entry Into the Distant Tail Lobes: ISEE-3, Geophys. Res. Lett., 11, 1078, 1984.

Harris, E. G.: On a plasma sheet separating regions of oppositely directed magnetic fields, Nuovo Cim., 23, 115, 1962.

Hesse, M., Birn, J., and Kuznetsova, M.: Collisionless Magnetic Reconnection: Electron Processes and Transport Modeling, J. Geophys. Res., 106, 3721, 2001a.

Hesse, M., Kuznetsova, M., and Birn, J.: Particle-in-cell simulations of three-dimensional collisionless magnetic reconnection, J. Geophys. Res., 106, 29 831, 2001.

Hesse, M., Kuznetsova, M., and Hoshino, M.: The structure of the dissipation region for component reconnection, Geophys. Res. Lett., 29(12), 1563, 2002.

Hesse, M., Winske, D., and Birn, J.: On the ion scale structure of thin current sheets in the magnetotail, Phys. Scr., T74, 63, 1997.

Hesse, M., Birn, J., Baker, D. N., and Slavin, J. A.: MHD Simulations of the Transition of Magnetic Reconnection from Closed to Open Field Lines, J. Geophys. Res., 101, 10 805, 1996.

Hesse, M.: The magnetotail's role in magnetospheric dynamics: Engine or exhaust pipe, US National Report to International Union of Geodesy and Geophysics 1991-1994, Rev. Geophys. Suppl., 675, 1995.

Hesse, M. and Winske, D.: Hybrid simulations of collisionless reconnection in current sheets, J. Geophys. Res., 99, 11 177, 1994.

Hesse, M., Schindler, K., Birn, J., and Kuznetsova, M.: The dif- 
fusion region in collisionless magnetic reconnection, Phys. Plasmas, 6, 1781, 1999.

Hones, Jr., E. W.: Substorm processes in the magnetotail: Comments on "On hot tenuous plasma fireballs and boundary layers in the earth's magnetotail” by Frank, L. A., Ackerson, K. L., and Lepping, R. P., J. Geophys. Res., 82, 5633, 1977.

Horiuchi, R. and Sato, T.: Particle simulation study of driven magnetic reconnection in a collisionless plasma, Phys. Plasmas, 1, 3587, 1994.

Hoshino, M., Nishida, A., Mukai, T., Saito, Y., Yamamoto, T., and Kokubun, S.: Structure of plasma sheet in magnetotail: Doublepeaked electric current sheet, J. Geophys. Res., 101, 24775 , 1996.

Le, G., Raeder, J., Russell, C. T., Lu, G., Petrinec, S. M., and Mozer, F. S.: Polar cusp and vicinity under strongly northward IMF on April 11, 1997: Observations and MHD simulations, J. Geophys. Res., 106, 21 083, 2001.

Machida, S., Ieda, A., Mukai, T., Saito, Y., and Nishida, A.: Statistical visualization of Earth's magnetotail during substorms by means of multidimensional superposed epoch analysis with Geotail data, J. Geophys. Res., 105, A11, 25 291-25 304, 2000.

Mandt, M. E., Denton, R. E., and Drake, J. F.: Transition to whistler mediated magnetic reconnection, Geophys. Res. Lett., 21, 73, 1994.

Mozer, F. S., Bale, S. D., and Phan, T. D.: Evidence of Diffusion Regions at a Subsolar Magnetopause Crossing, Phys. Rev. Lett., 89, $15002,2002$.

Nagai, T., Shinohara, I., Fujimoto, M., Hoshino, M., Saito, Y., Machida, S., and Mukai, T.: Geotail observations of the Hall current system: Evidence of magnetic reconnection in the magnetotail, J. Geophys. Res., 106, A11, November 1, 25 929-25 950, 2001.

Nagai, T., Fujimoto, M., Nakamura, R., Saito, Y., Mukai, T., Yamamoto, T., Nishida, A., Kokubun, S., Reeves, G. D., and Lepping, R. P.: Geotail observations of a fast tailward flow at $X_{G S M}=-15 R_{E}$, J. Geophys. Res., 103, A10, 23 543-23 550, 1998.

Øieroset, M., Phan, T. D., Fujimoto, M., Lin, R. P., and Lepping, R. P.: In situ detection of collisionless reconnection in the Earth's magnetotail, Nature, 6845, 414, 2001.

Perreault, P. and Akasofu, S.-I.: A study of geomagnetic storms, Geophys. J. R. Astr. Soc., 54, 547, 1978.
Priest, E. R.: Solar Flare Magnetohydrodynamics, Gordon and Breach, New York, 1981.

Priest, E. R. and Forbes, T. G.: The magnetic nature of solar flares, Astron. and Astrophys. Rev., 10, 313, 2002.

Pritchett, P. L.: Geospace Environment Modeling magnetic reconnection challenge: Simulations with a full particle electromagnetic code, J. Geophys. Res., 106, 3783, 2001.

Pulkkinen, T. I., Baker, D. N., Fairfield, D. H., Pellinen, R. J., Murphree, J. S., Elphinstone, R. D., McPherron, R. L., Fennel, J. F., and Lopez, R. E.: Modeling of the growth phase of a substorm using the Tsyganenko model and multi-spacecraft observations, Geophys. Res. Lett., 18, 1963, 1991.

Russell, C. T. and Elphic, R. C.: ISEE observations of flux transfer events at the dayside magnetopause, Geophys. Res. Lett., 6, 33, 1979.

Sanny, J., McPherron, R. L., Russell, C. T., Baker, D. N., Pulkkinen, T. I., and Nishida, A.: Growth phase thinning of the near-Earth current sheet during the CDAW 6 substorm, J. Geophys. Res. 99, 5805, 1994.

Schindler, K., Hesse, M., and Birn, J.: Magnetic- field-aligned electric potentials in non-line-conserving flows, Astrophys. J., 380, 293, 1991.

Sonnerup, B. U. O., Paschmann, G., Papamastorakis, I., Sckopke, N., Haerendel, G., Bame, S. J., Asbridge, J. R., Gosling, J. T., and Russell, C. T.: Evidence for magnetic field reconnection of the earth's magnetopause, J. Geophys. Res., 86, 10 049, 1981.

Sonnerup, B. U. O., Paschmann, G., Papamastorakis, I., Sckopke, N., Haerendel, G., Bame, S. J., Asbridge, J. R., Gosling, J. T., and Russell, C. T.: Evidence for magnetic field reconnection of the earth's magnetopause, J. Geophys. Res., 86, 10 049, 1981.

Vasyliunas, V. M.: Theoretical models of magnetic field line merging. I, Rev. Geophys. Spac. Phys., 13, 303, 1975.

Yamada, M., Ji, H., Hsu, S., Carter, T., Kulsrud, R., Ono, Y., and Perkins, F.: Identification of Y-shaped and O-shaped diffusion regions during magnetic reconnection in a laboratory plasma, Phys. Rev. Letts., 78, 3117, 1997.

Zeiler, A., Biskamp, D., Drake, J. F., Rogers, B. N., Shay, M. A., and Scholer, M.: Three-dimensional particle simulations of collisionless magnetic reconnection, J. Geophys. Res., 107, 1230, 2002 . 